\title{
The universal, collaborative and dynamic model of specialist and advanced nursing and midwifery practice: A way forward?
}

Laserina O'Connor

Mary Casey

University College Dublin, Ireland

Rita Smith

University College Dublin, Ireland

See next page for additional authors

Follow this and additional works at: https://arrow.tudublin.ie/tfschhmtart

Part of the Health Policy Commons

\section{Recommended Citation}

O'Connor L. et al. (2018) The universal, collaborative and dynamic model of specialist and advanced nursing and midwifery practice: A way forward? Journal of Clinical Nursing, 27, e882-e894. doi: 10.1111/ jocn.13964

This Article is brought to you for free and open access by the School of Tourism \& Hospitality Management at ARROW@TU Dublin. It has been accepted for inclusion in Articles by an authorized administrator of ARROW@TU

Dublin. For more information, please contact

arrow.admin@tudublin.ie, aisling.coyne@tudublin.ie, gerard.connolly@tudublin.ie.

Funder: Nursing and Midwifery Board of Ireland

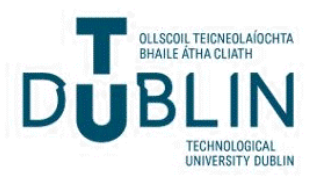




\section{Authors}

Laserina O'Connor, Mary Casey, Rita Smith, Gerard M. Fealy, Denise O'Brien, Denise O'Leary, Diarmuid Stokes, Martin S. McNamara, Mary Ellen Glasgow, and Andrew Cashin 


\title{
The universal, collaborative and dynamic model of specialist and advanced nursing and midwifery practice: A way forward?
}

\author{
Laserina O'Connor, Mary Casey, Rita Smith, Gerard M Fealy, Denise O' Brien, Denise O'Leary, \\ Diarmuid Stokes, Martin S McNamara, Mary Ellen Glasgow, Andrew Cashin \\ Citation: O'Connor L., Casey M., Smith R., Fealy G.M., O'Brien D., O'Leary D., Stokes D., \\ McNamara M., Glasgow M.E., Cashin A. (2018) The universal, collaborative and dynamic model of \\ specialist and advanced nursing and midwifery practice: A way forward? Journal of Clinical \\ Nursing, 27, e882-e894.
}

\section{ABSTRACT}

Aims and objectives

To inform and guide the development of a future model of specialist and advanced nursing and midwifery practice.

\section{Background}

There is a sizable body of empirical literature supporting the unique contributions of specialist and advanced practice roles to health care. However, there is very little international evidence to inform the integration of a future model for advanced or specialist practice in the Irish healthcare system.

Design

A qualitative study was conducted to initiate this important area of inquiry.

Methods

Purposive sampling was used to generate a sample of informants $(n=15)$ for the interviews. Nurses and midwives working in specialist and advanced practice and participants from other areas such as legislative, regulatory, policy, medicine and education were included in the sampling frame.

Results

Arguments for a new model of specialist and advanced practice were voiced. A number of participants proposed that flexibility within specialist and advanced practitioner career pathways was essential. Otherwise, there existed the possibility of being directed into specialised "silos," precluding movement to another area of integrated practice. Future specialist and advanced practice education programmes need to include topics such as the development of emotional and political intelligence.

\section{Conclusion}

The contribution of specialist and advanced practice roles to the health service includes providing rapid access to care, seamless patient flow across services, early discharge and lead coordinator of the patient's care trajectory. There was a recommendation of moving towards a universal model to cultivate specialist and advanced nurse and midwife practitioners. 
The model design has Universal application in a range of contexts " $\mathrm{U}$." It is Collaborative in its inclusivity of all key stakeholders " $\mathrm{C}$." The model is Dynamic pertinent to accommodating movement of nurses and midwives across health continua rather than plateauing in very specialised "silos" "D."

What does this paper contribute to the wider global clinical community

The Universal, Collaborative and Dynamic Model for specialist and advanced nurse/midwife practitioners embraces capability as a concept that emphasises lifelong learning in uncertain as well as familiar intricate clinical situations.

The Universal, Collaborative and Dynamic Model recommends that the measurement of patientreported outcomes, clinical outcomes and health economic outcomes by specialist and advanced nurse/midwife practitioners is required for accountability, that is, performance indicators that are consistent with professional knowledge.

The model design has Universal application in a range of contexts "U." It is Collaborative in its inclusivity of all key stakeholders "C." The model is Dynamic relevant to enabling nurses and midwives to transition their clinical and professional roles across the health continuum " $D$," with a person-centred ethos at its core.

\section{INTRODUCTION}

There is a sizable body of empirical literature supporting the unique contributions of specialist and advanced practice nursing and midwifery to health care. However, there is very little international evidence to inform the integration of a future model for advanced or specialist practice in the Irish healthcare system. Indeed, what has become apparent is the dearth of available evidence pertaining to the use of and validation of conceptual models developed to guide advanced and specialist nursing and midwifery practice. The purpose of this study was to inform and guide the development of a future model of specialist and advanced nursing and midwifery practice, taking emerging and future service needs into account.

\section{BACKGROUND}

Any model proposed to guide future service needs must include provision for capturing clinical outcomes. This approach ensures that any healthcare delivery is keeping pace with the demands of a constantly changing context. This is important as the conceptual deficiencies of previous research relate to the lack of a theoretical framework guiding research, and the inability to differentiate outcomes of care pertinent to nursing and midwifery practitioners from other healthcare providers (Dowling, Beauchesne, Farrelly, \& Murphy, 2013; Elliott et al., 2013; Sidani \& Irvine, 1999; Stasa, Cashin, Buckley, \& Donoghue, 2014).

The model that is introduced must be guided by principles of inclusion and participation, meaning it must be operationalised using a collaborative process (Cashin, 2014; Stasa et al., 2014). In addition, the model should facilitate an accurate measurement of clinical outcomes. As a starting point, this means that the core activities of these roles for nursing and midwifery are described in a relevant manner and are transferable into service provision. The model proposed therefore needs to be contextually sensitive, hence the impetus for this study.

\section{METHODS}




\subsection{Study design}

The authors conducted a qualitative study, to initiate an important area of inquiry for specialist and advanced nursing and midwifery practice. Accordingly, a rapid review of the literature was undertaken and reported in detail elsewhere (O'Leary et al., 2017). Semistructured interviews were undertaken with key stakeholders (Table 2), to ascertain their views on current and possible future models of specialist and advanced nursing and midwifery practice. This approach was taken because it permits an in-depth exploration of the phenomena in question and takes culture, context and sociopolitical factors into account (Cresswell, 2014). This type of approach is particularly useful in nursing research, as the philosophy underpinning qualitative research is compatible with nursing philosophy (Parahoo, 2014). This allowed specialist nurses and midwives and advanced nurse and midwife practitioners and key stakeholders $(n=15)$ to provide individual feedback on all aspects of the topics under review.

The interview aims were to:

- explore the views of participants on specialist and advanced nursing and midwifery practice, taking into account the outcomes and impact of practice in relation to quality of care, cost and access to services;

- elucidate participants view on current and possible future models of specialist and advanced nursing and midwifery practice taking into account emerging and future service needs; and

- explore methods for capturing specific clinical outcomes for specialist and advanced practice interventions for their clinical speciality.

\subsection{Sample details and recruitment methods}

Prior to recruitment into the study, a range of eligible key stakeholders $(n=15)$ were informed about the study by letter. This approach was taken to gain a multifaceted perspective on the research questions in an Irish context. Purposive sampling was used to generate this sample of informants for the interviews. Nurses and midwives working in specialist and advanced practice, as well as participants from other key areas such as legislative, regulatory, policy, medicine and education, with a wealth of knowledge on the topics under scrutiny, were included in the sampling frame. All key stakeholders $(n=15)$ agreed to participate in the study.

\subsection{Participants}

A demographic profile of participants is presented in Table 1 . The majority of participants were over the age of 40 years and female. Participants were all well-educated, with the majority having a master's-level qualification. The majority of participants were in their roles for more than 1 year.

Insert Tables 1 \& 2 here

As planned, participants were engaged in a range of different roles as highlighted in Table 2 Approximately half of the participants were either specialist nurses or midwives or advanced practitioners. To maintain anonymity, each participant is identified by a unique code (Table 2 ).

\subsection{Data collection and conduct of interviews}

Semistructured interviews lasted between 60-90 min. An interview topic guide with open-ended questions, supported by a series of probes, was used to encourage a range of responses. The 
interviewers were mindful that interviews are one of the most common and powerful ways in which we try to understand the phenomena of interest. Therefore, every effort was made to undertake the interviews in locations where participants felt comfortable to share their views and experiences, that is, employment settings. The interview topic guide (Appendix S1) was subjected to pilot testing initially at the level of the research team, and no modifications were made.

\subsection{Ethical considerations}

The study was approved by the Human Research Ethics Committee at University College Dublin. Principles of good practice with regard to ethics were followed during the study. A formal letter, an information leaflet detailing the aim of the study, and a consent form were provided for each potential participant. All participants signed a written consent form before being interviewed. They had the option of withdrawing from the study at any stage. All data were anonymised and pseudonyms were used when discussing the findings.

\subsection{Data analysis}

All interviews were audio-recorded with the permission of participants and transcribed verbatim. Data analysis was guided by the constant comparative technique (Corbin \& Strauss, 2008). This ensured that all data were systematically compared to all other data, to enable the recognition of emerging and embedded themes. In this way, data from subsequent coding process influenced recoding of the first participants data and the consequent coding of the remaining participants' data. Two members of the research team analysed the same transcript and agreed a coding framework. The agreed coding framework was used to code the remaining interviews. The initial open codes broke the data down into smaller units of analysis. During axial coding, these codes were collapsed into categories. These categories were compared using selective coding. This process resulted in the clustering and collapsing of codes, and the final identification of themes (Strauss \& Corbin, 1998).

NVIVO version 11, qualitative data research software, was used to organise, explore and manage the data (QSR International). The focus of the analysis was on both description and the interpretation of the phenomena of interest. Coding reached theoretical saturation when no new themes emerged. The criteria used for assessing the truth value of this study were operationalised in various ways. These included maintaining a chain of evidence and providing rich accounts so that the reader feels as if she/he were present, referred to as naturalistic generalisation (Stake \& Trumbull, 1982). The identification of themes evolved from the analysis of the narrative, which is outlined in the Results section.

\section{RESULTS}

A total of 15 key stakeholders were invited and participated in the semistructured interviews. The data were analysed thematically into themes. Two subthemes are enclosed by the larger theme of impact of specialist and advanced practice roles. The first theme was the "contribution of the nurse specialist (NS), midwife specialist (MS), advanced nurse practitioner (ANP), and advanced midwife practitioner (AMP) role in the health service/system." The second theme noted was the "advanced and specialist nurses and midwives enhance patient care."

\subsection{Contribution of the NS/MS/ANP/AMP role in the health service/system}

In general, there was agreement among participants that specialist and advanced practitioners made a valuable contribution to the health service. It seemed that the uniqueness of their knowledge and skills appeared to provide better patient outcomes and was more cost-efficient. It appeared that managing an entire episode of care was inherent in their autonomous role. This model was 
highlighted as beneficial, as it allowed nurses and midwives to interact with patients/clients at all stages of their care. It was also about the delivery of a more holistic model of care from admission to discharge:

...somebody working from an autonomous level...it is a model... you are involved in the whole process of seeing the patient, or the woman, or the client, diagnosing, prescribing and discharge where sometimes you are cutting out the medical aspect of it... so it is a holistic aspect from a nursing or midwifery background. (AP4)

Potential cost saving was highlighted by participants pertinent to hospital avoidance, suggesting a role in the community for ANPs which would "replace hospital appointments which would free up the hospital resources then, to deal with other issues" (MPNRB1). Financial consideration of the impact of the role of the ANP was also mentioned. This was voiced in the context of impact on patient outcomes in a community pharmacy placement, "it is a cheaper and better way of delivering the service. There is a cost for people coming into hospitals. There is the patient convenience, which is an important issue, and there is also the clinical outcomes" (MPNRB1).

\subsection{Advanced and specialist nurses and midwives enhance patient care}

In this subtheme, there was a suggestion that a particular strength of the ANP role in the acute setting is to be able to see both the medical and nursing aspects of care. The ANP takes a more holistic approach that essentially brings the diagnosis and caring aspect of health care together in patient outcomes:

In terms of outcome our knowledge, our experience in dealing with the sicker baby...our ability to stand back and see, from a nursing point of view, what makes a contribution to the babies care...we see....because we have one leg straddling each camp...we can see how we can marry the nursing actions with the medical diagnosis and the medical care that the baby is receiving in a way that the consultant and the registrars mightn't necessarily see. (AP1)

\subsection{Interdisciplinary interactions}

One major subtheme that emerged was related to working with the medical staff in an interdisciplinary context in the acute setting.

Advanced midwife practitioner/ANP participants gave an account of interdisciplinary caseload management. This tactic demands a willingness to work in a very flexible manner, depending on the needs of a particular situation. It suggests a high level of autonomy on the part of the AMP/ANP. That said, it also concerns decision-making and a supportive role to nursing and medical colleagues in the provision of acute care: "...if there is a high risk delivery...making sure we attend that delivery...if there is a really sick baby we would spend the day in the unit supporting both the nursing staff and the doctor that's on call" (AP1).

As advanced midwife practitioners (AMPs) were able to see women early in gestation, an opportunity to engage in shared care in an interdisciplinary context with the obstetrician was highlighted:

...to take on another patient, this is where the growth of the role comes in, this is where the motivation comes in, I would present it to...using the ISBAR tool to my obstetrician...full assessment process, why I would like to take on this patient....but it is sort of shared care so she is getting that whole midwifery input but she is under the umbrella of an obstetrician also. That is interdisciplinary working at its best... I will take the case but I will make sure that my decision making and my record 
keeping and the evidence behind the reason I am taking on that woman are quite robust, that is where the role comes in. (AP4)

The centrality of the role of the ANP was highlighted as being the health professional who, in addition to their own contribution to care, essentially, was the lead coordinator of the patient's care trajectory:

They [referring to ANPs] are the central piece to ensuring the patient's journey across all of those, either they have their own piece of the jigsaw to do, but they are often the centre piece that the consultant comes back to, that pathology comes back to, the radiologist comes back to... so they are the piece that kind of seals up a multidisciplinary team as a central conduit for all the patient information...and not only that but they have the expertise to be able to suggest to the consultant whatever. (MPNRB3)

In most cases, collaboration and teamwork were terms used interchangeably across settings. However, to extrapolate discipline-specific outcomes on integrated care teams was identified as a challenge.

\subsection{Impact of specialist and advanced practice roles on patient outcomes}

The two subthemes that emerged were (i) measuring impact of specialist and advanced practice roles and (ii) quality, safety and patient access to comprehensive care.

\subsubsection{Measuring impact of specialist and advanced practice roles}

The challenges and difficulties of attributing direct impact on patient outcomes to a specific medical or nursing or midwifery role are well known. The participants identified that team-based care approaches add another level of complexity: "it is very difficult to measure what influence they have because it's a team approach...so the team cares for each baby...so the actual individual contribution of each team member is actually difficult to measure" (AP1).

In terms of impact on patient outcomes, the role of the ANP in the community-based pharmacy appeared to have a positive impact. This effect was highlighted in the context of chronic disease management:

The patients were dealt with in a very emotionally intelligent way and it managed to persuade them that it was in their best interest to introduce certain changes in their life style or their approach to diabetes...things like not injecting in the same site continuously where depots of insulin were deposited in the body... they learned how to do it properly and that problem was rectified. (MPNRB1)

There was almost tangible evidence of the impact of NS roles on patient outcomes, pertinent to chronic disease management, and hospital avoidance. For example, patient flow was seen as seamless and in particular if the NS was also a prescriber:

Whereas in the nurse led clinic, we see the patients who are having eye problems and we do a full thorough assessment, spend time with them, initiate treatment that is warranted and follow up with them....and that is a huge aspect of work, trying to keep people away from the hospitals, safe at home and keep them out of ED. (CS1)

\subsubsection{Quality, safety and patient access to comprehensive care}

In terms of quality and safety, good work practices in the acute setting can reduce length of stay and promote early discharge. From a midwifery perspective, quality and safety of care was related to 
"...so we have got a high normal delivery rate, it means average length of stay is decreased...women get to debrief with the midwife quicker so at the end of the day they are not spending time in hospital...they are getting home." (AP4)

In terms of community care and nursing home care, the medical perspective on the quality and safety aspect acknowledged that ANPs can intervene in an anticipatory and protective role:

The advanced nurse practitioner would manage a significant percentage of the work which perhaps the nursing home itself might refer to them. Often it's very simple things; it's medication adjustment, recognising that...you know...constipation can be caused by dehydration, it can be caused by lack of mobility...you know...so you need to address these things rather than just prescribing a drug which often is the default position of the busy locum doctor coming in and start them on something and then gone again as opposed to engaging... (MPNRB2)

In some instances, particularly in midwifery care contexts, access to care is easy as highlighted in the following excerpt:

They [referring to patients] can pick up the phone... the AMP have five referral pathways, obstetricians, midwives, clinical governance, the GP, and the woman herself...so in a nutshell...GPs ringing in for women to attend the advanced midwife practitioner service...access is very, very easy. (AP4)

Patient access to comprehensive chronic illness care was also highlighted by patients from the community pharmacist, associated with having an ANP based in the community pharmacy on a regular basis.

\section{FUTURE DEVELOPMENT OF NS/MS/ANP/AMP ROLES FOR CONSISTENCY}

Four subthemes are covered by the larger theme of future development of NS/MS/ANP/AMP roles. These are (i) realising the potential of specialist and advanced practice NS/MS/ANP/AMP roles, (ii) defined career pathway, benchmarks and performance indicators, (iii) research activities and postgraduate education and (iv) future models of specialist and advanced practice roles.

\subsection{Realising the potential of specialist and advanced practice roles}

All participants agreed that specialist and advanced practice roles provided added value to the health service, and had the potential to add even more value. This value added relates to access to care, patient flow, early discharge, hospital avoidance and case management. There was recognition of the current changes to the health system with increasing focus on primary and community care sectors. Participants regarded these patterns of service delivery as opportunities for specialist and advanced nurses and midwives, to develop and implement new types of care delivery systems.

Practice nurses and ANPs were ideally positioned as having the potential to address a growing need in primary care, and to complement the work of general practitioners (GPs). However, participants were clear that nurses in these roles should be positioned as nurses rather than GP substitutes. Therefore, nursing discipline boundaries and scope of practice are acknowledged as distinct and valid, “...[advanced nurse practitioners] with a knowledge and a skill at a level within their own profession and within their own scope that's just as valid and just as valuable to the care of the patient" (AP1).

\subsection{Defined career pathway, benchmarks and performance indicators}


A number of participants suggested that without flexibility in a career pathway, there existed the possibility of being directed into very specialised "silos," precluding movement to another area of practice. A common subject running through participants' views of models of specialist and advanced practice was the acknowledgement that patients, and their health needs, should be at the epicentre of any future plans for development and expansion:

Opportunities exist for nurses to practice at advanced level for the benefit of patients not for the benefit of purely advancing nursing or midwifery. The end point is providing advanced care for patients and achieving that aim for the population. (AP1)

Participants argued that nurses and midwives who have been in specialist and advanced positions for a number of years should supervise others in training for these roles, "why can't AMPs supervise other AMPs... why does it have to be an obstetrician now going forward to grow other people...this is a resource that has yet to be tapped and supervision could be introduced as an element of a future model" (AP4).

Key performance indicators were suggested as one possible addition to a model and could be a way of defining goals and evaluating performance.

\subsection{Research activities and postgraduate education}

Engagement with the research agenda was seen as a challenge for most participants, at both specialist and advanced level. Some suggestion of establishing communities of knowledge was mentioned as a possible option. Currently, ANPs and AMPs are expected to engage in research as part of their role. However, there was a sense that the research element was secondary to the clinical element, "definitely the main part of my work is clinical and I think that's the way it should be to be honest" (AP3).

Participants from the practice and policy arenas were in agreement that due to clinical commitment issues, it is unrealistic to expect either a specialist or an ANP or AMP to engage in research on their own. More informal linkages were mentioned by participants who described helpful interactions with academics. Other participants suggested that being part of a multidisciplinary clinical team involved in research was a way to address their research agenda. Indeed, it was argued that healthcare-related research should be multidisciplinary in nature and "doesn't just have to be about the nursing bit" (PRME4).

There was general agreement that the current educational preparation for ANP and AMP roles in Ireland requires a review. This was placed in the context of the 4-year undergraduate degree programme in Ireland, which was seen as providing a solid foundation for their career pathway:

It sounds a particularly long time [the current 7 year post registration requirement for ANP]... I think the whole piece needs to be looked... because...I just think now with the newer graduates there is a different breed who are at an honours degree level and really we have to ensure safety, we have to ensure that they are competent, we have to ensure that they have the skills and ability to do the job...but as well, we have to give them the scope in order to evolve and expand if they should choose to go on that pathway. (MPNRB3)

\subsection{Future models of specialist and advanced practice}

Arguments for a new model of specialist and advanced practice were voiced, with a more universal approach to advanced practice. In terms of future models, the benefits of continuing with a portfolio of evidence were suggested, "the portfolio is good" (AP1), and a more structured approach to 
experiential learning, "...there needs to be some sort of mechanism of how we can actually recognise or put a quality stamp on some of these education bites... so that people are developing these baskets of learning and baskets of skill sets that are unique to them and to streamline and make that easy for people..." (PRME1).

All participants reported that regardless of what models are put forward, the role of education and clinical experience in shaping the future of these roles is really important:

There is nothing like experience except for it does need to be hand in hand with education and I think it is the education piece that really challenges us and puts us into creative thinking people because like all of us we can get into a culture of you know your business so well but I think experience is really important. (MPNRB3)

All were in agreement that content for future specialist and advanced practice education should include topics such as self-development, self-awareness and developing emotional and political intelligence.

\section{DISCUSSION}

The contribution of specialist and advanced practice roles to the health service included providing rapid access to care, coordinating interdisciplinary team care and a more holistic model of care. The views of policymakers on the impact of specialist and ANP/AMP practice roles in Ireland were explored in the SCAPE study (Begley, Murphy, Higgins, \& Cooney, 2014). They found that ANPs provided more collective care links for broadening the vision and concept of holistic care. This has implications for how competency is developed and whether the development is linear at specialist level or multidirectional at ANP level (Begley et al., 2014).

In this study, specialist and advanced practice roles function well within an interdisciplinary team context. The ability of the ANP/AMP to straddle both a nursing and medical boundary provided a lens into the quality of care delivered from a team perspective. However, the difficulty in separating the NS/MS/ANP/AMP/contribution to patient outcomes from that of the other multidisciplinary team members was highlighted. The attribution of the ANP to specific outcomes was found to be very messy to extrapolate because of the complexity of the intervention, and multiple team members (Stanik-Hutt et al., 2013). Measuring outcomes and contributions to care is an essential element of their use, and the effects of care need to be captured through measurement (Maben, Morrow, Ball, Robert, \& Griffiths, 2012). In this study, reference was made to how care is carried out by the ANP against a standard.

Ease of access to patients was underlined as another method of enhancing patient care. In this study, access to multidisciplinary care in the context of chronic illness and underserved community populations was reported. According to McClellan, McKethan, Lewis, Roski, and Fisher (2010), access to care should be reflected within a multidisciplinary model of care and not reflective of a onedimensional nurse-led or midwife-led service. Access to diabetes care via a community pharmacy experience was identified as positive by participants with a quality, and access outcome, as well as reduced costs by freeing up hospital resources. This finding is important and highlights that ANPs can provide a complimentary service to other members of the multidisciplinary team in underserved populations. Everett et al. (2013) found that nurse practitioners can successfully fill a range of roles on the primary care team, and with this team-based approach to care can improve access, and reduce costs in the context of diabetes care. 
There was general agreement in this study, about an untapped potential in specialist and advanced practitioner services, which could be expanded to the community and primary care sectors. These services relate to access to comprehensive care, patient flow, hospital avoidance and early discharge from the acute care setting to home. Furthermore, the ability of the nurse and midwife to prescribe medicinal products for patients was seen as important in ensuring the delivery of optimal and costeffective outcomes across all care services. Researchers examined efficiency models for the provision of cost-effective nurse practitioner service in primary care settings (Liu \& D'Aunno, 2012; Liu, Finkelstein, \& Poghosyan, 2014). They recommend that healthcare organisations should focus on better using the ANP role to contain costs, and improve access to care. According to Martin-Misener et al. (2015), measuring the cost-effectiveness of ANPs in alternative and complimentary roles has never been greater, as the consequences of chronic disease continue to be revealed.

Participants highlighted that a structured career pathway is needed and must take client or patient needs into account. Benchmarks and key performance indicators were suggested as useful additions to such a career pathway to enable nurses and midwives to interpret their progress. ANP/AMP and NS/MS undertake an increasing role in providing care to patients across a diverse range of settings. Therefore, measuring the impact of their care on patient outcomes and quality of care measures becomes a necessary constituent of performance evaluation. The important element of this process is to align nursing and midwifery metrics with what matters most to patients, to staff and across the organisation (Maben et al., 2012). Moreover, Kilpatrick et al. (2014) reported that objective performance measures are needed for a full assessment of the cost-effectiveness of the specialist and advanced practice role, and to systematically monitor quality and outcomes of care in the context of chronic illness.

Supervision by midwives, who have been in specialist and advanced positions for a number of years, was identified as an untapped resource and ought to be introduced within a future model of specialist and advanced practice. Poghosyan et al. (2013) reported on the elements that either supported or restricted the NP's scope of practice: the regulatory environment as reflected in the requirement to have physician supervision in nurse prescribing, and physician collaboration and support, whereas Cashin et al. (2015) referred to nurse practitioners as educators meaning role models and mentors to nurses and health professionals.

There were mixed views on the need for research as an integral element of the advanced practitioner role. A number of participants felt that expecting nurses and midwives in these roles to undertake independent research was unreasonable due to clinical activities. Only a small minority suggested that engaging in independent research was achievable and an important component of the advanced practice role. The findings support previously reported data (Begley et al., 2010; Gerrish et al., 2012, O'Keeffe, Corry, \& Moser, 2015; RCSI, 2010; Wilkes, Mannix, \& Jackson, 2013). In this study, there was openness to engaging in research in partnership with academics or a multidisciplinary team. O'Keeffe et al. (2015) proposed that ring-fencing of research hours should be targeted as a key area to be addressed, in the immediate future for specialist and advanced practitioners.

In this study, all participants suggested that content for future education should include topics such as self-development, self-awareness and developing emotional and political intelligence. Some discussion focused on continued professional development with practitioners maintaining a portfolio of evidence and participating in relevant "baskets of learning." The Nursing Midwifery Board of Ireland identified the "applicant's portfolio" as part of the criteria for registration to enter the Advanced Practice Division of the Register (NMBI, 2017). 
There was also a suggestion of moving towards a more universal model, to prepare advanced nurse and midwife practitioners. A number of participants suggested that without flexibility in a career pathway, there existed the possibility of being directed into very specialised "silos." This approach restricted movement to another area of practice, rather than having a broader area of specialisation within which there is career mobility. Cashin et al. (2015) found that nurse practitioners in Australia, rather than moving further in the same direction of specialisation, become more generalist.

\section{LIMITATIONS}

While a wide range of views were received from the 15 participants, the majority of participants were specialist and advanced nurse and midwifery practitioners employed in large university hospitals. The views of staff nurses and staff midwives, public and patient groups, and staff in education were not well represented in these data.

The research design and associated data collection methods were well supported in the literature. However, the small sample of participants and the use of nonprobability sampling methods can be prone to sample bias, which could reduce the study's population validity.

Nevertheless, the study does make some attempt to provide direction in terms of a suitable model for specialist and advanced nursing and midwifery practice. A brief discussion of this model is outlined hereunder.

\section{DISCUSSION ON THE MODEL}

This model represents a progressive pathway for nurses and midwives from graduate to doctoral level. The evolution of the Universal, Collaborative and Dynamic Model of specialist and advanced nursing and midwifery practice is based on the evidence-based review (Casey et al., 2015) and the qualitative findings. In addition, elements of a conceptual framework developed by (i) Maben et al. (2012), (ii) the Transformational Advanced Practice Provider Model (Elliott \& Walden, 2014) and (iii) the framework put forward by Gerrish, McDonnell, and Kennedy (2013) were considered. There is also both a practice and educational and continuous professional development aspect to the model. This model has Universal application in a range of contexts " $U$ "; is Collaborative in its inclusivity of all key stakeholders " $\mathrm{C}$ "; and is Dynamic in the context of transition within three continua and alignment of the six core concepts "D."

There are four elements to the Universal, Collaborative and Dynamic Model of specialist and advanced nursing and midwifery practice. These are (i) six concepts of professional and clinical practice with person-centred care as the core concept, (ii) three continua that reflect the professional practice and development trajectory, (iii) key performance indicators within a metrics schema for both specialist and advanced practice and (iv) an iterative framework schema to evaluate the impact of such roles.

\subsection{Core concepts}

Diagrammatically five concepts, accountability, professional ethics, consultation and collaboration, professional leadership and clinical scholarship, are exemplified as triangles that merge on a central core concept, that is, person-centred care. The entire circle that embraces the six core concepts represents the fluidity of each of the core elements that are always in a state of development. A brief overview of each core concept is provided below.

Person-centred care 
The central placement of "person-centred care" highlights the value placed on what matters to the patient in their care trajectory. This concept gives consideration to the patient's clinical status, desires, values, family situations, social circumstances and lifestyles. It also reflects seeing the person as an individual, and working together to develop appropriate solutions (Greene, Tuzzio, \& Cherkin, 2012; Nardi \& Diallo, 2014; Scholl, Zil, Harter, \& Dimaier, 2014). Person-centred care is about forensically unravelling each patient's episode of care across a healthcare continuum.

\section{Accountability}

Accountability enables the specialist or advanced nurse/midwife practitioner to practise with authority, autonomy and responsibility. This translates to act within an interdisciplinary team context across services, with a nurse/midwife-led agenda of quality improvement and safe positive patient outcomes. In 2001, the Institute of Medicine recommended that we move away from single providers and single disciplines working as islands unto themselves. Accordingly, integrated care teams need to have authority and accountability to maximise the contributions of each practitioner towards system goals that benefit the patient and society (Newhouse et al., 2012).

\section{Professional ethics}

This concept relates to the individual and organisational standards of professional behaviours, expected of the specialist and advanced nurse/midwife practitioner. This means being at the helm of a just culture healthcare environment. Therefore, the setting is navigated by both transparency and accountability, and one that supports improved patient and organisational outcomes.

\section{Consultation and collaboration}

The concept of consultation and collaboration is crucial to high-quality care. Future healthcare workforce policy must reject practice "silos" (Newhouse et al., 2012). As we embrace integrated models of healthcare delivery, healthcare professionals work in partnership across disciplinary boundaries to produce new and more effective models of care. In an integrated care approach pertinent to this model, physicians refer patients to the specialist nurse or midwife/advanced practitioner with expertise in managing acute and/or chronic care populations and vice versa.

\section{Professional leadership}

The specialist or advanced nurse/midwife practitioner is involved with disseminating knowledge about the impact of his/her role, and influencing health and public policy. They use systems insight to engage major stakeholders, in evaluation of care practices that lead to organisational efficiencies and improved outcomes for the patient, staff and organisation (Maben et al., 2012).

\section{Clinical scholarship}

Clinical scholarship is defined by Palmer (1986) as the integration of theoretical and experiential knowledge, derived from the analytical observation of clients and patients. Palmer highlights that it must include intellectual activity of thinking, analysis and synthesis. Diers (1995) extends this definition to include writing - the critical dissemination stage of generating knowledge. This concept within the model is about a culture of clinical inquiry. Such a culture empowers specialist and advanced practitioners to think outside the box, and to do, and then to disseminate research universally.

\subsection{Professional and clinical practice continua}


Three continua set the clinical context for this model, namely the professional and clinical continuum, the health continuum and the role continuum. The professional and clinical continuum reflects a trajectory of growth that never plateaus. On the role continuum, the specialist and advanced nurse/midwife practitioner construct their own learning outcomes, reflect and fuse many sources of data related to modalities of practice and role responsibilities that oscillate over time.

The health continuum builds on the principles of person-centred care. It includes the development of healthful relationships across health situations, and levels of unpredictable and more complex conditions. The specialist nurse/midwife will have a population-specific condition focus applicable to healthcare conditions. As transformation occurs, the health continuum as it relates to the advanced nurse/midwife practitioner will centre on universal healthcare needs within an integrated care model. The integrated care model approach will enable advanced nurse/midwife practitioners to deliver care in response to population health needs.

The Universal, Collaborative and Dynamic Model reflects the fluidity of each core concept, related to the level of engagement of the specialist and advanced practitioner with the three continua. This alignment sits within a competency and capability framework of continual higher-level learning. The professional and clinical continuum reflects the population and lifespan focus of the specialist role and further embraces capability as an approach for advanced practice and education. O'Connell, Gardner, and Coyer (2014) suggest there is a need to embrace capability as a framework for advanced practice and education. This need is described against the backdrop of viewing the complexity of the clinical environment, which is nonlinear. Capability was articulated by Cairns $(2000$, p. 1) as "...having justified confidence in your ability to take appropriate and effective action to formulate and solve problems in both familiar and unfamiliar and changing settings." Meanwhile, according to O'Connell et al. (2014), competency suggests a stable if somewhat static outcome because there are predesigned skills to be achieved. Adaptability to change and an emphasis on lifelong learning are thus central but are usually conspicuously absent in competency-based initiatives (Phelps, Hase, \& Ellis, 2005). Developing standards for advanced clinical learning, and teaching, and furthering understanding of capability theory for advanced healthcare practitioners will contribute to evidence-based models of advanced speciality education (Gardner, Gardner, Coyer, \& Gosby, 2016). The term capability is reflected in the very recent definition of "advanced nursing practice" by the Nursing Midwifery Board of Ireland (NMBI):

...a career pathway for registered nurses, committed to continuing professional development and clinical supervision, to practice at a higher level of capability as independent, autonomous, and expert practitioners. $(2017$, p. 15)

\subsection{Specialist and advanced key performance indicators}

Another specification of this model is specialist performance indicators, which indicate the first point of adaptability by the nurse/midwife to the specialist level. There is a strategic bidirectional relationship with the core concepts and the key performance indicators. As the specialist and advanced nurse/midwife practitioner assume an increasing role in providing care to patients across diverse settings, measuring the impact of their care on patient-related outcomes is a necessary constituent of their performance.

There is a reciprocal connection between the "quality and performance metrics evaluation" and the "core concepts and key performance indicators." This connection pertains to a measurement system that would enable the collection and analysis of data on three perspectives related to the impact of 
the specialist/advanced practitioner, that is, operational excellence, value proposition and economic value.

There is also an iterative relationship between both the specialist and advanced key performance indicators that envelop the six core concepts of professional and clinical practice and the three continua within zones of health complexity. The development of advanced key performance indicators with key stakeholders will enable the advanced practitioner to measure his/her actual direct and indirect impact on practice, across four high-level domains: clinical, professional, organisational (Gerrish et al., 2013) and the healthcare system.

\subsection{Quality and performance metrics}

Person-centred care is at the epicentre of the Universal, Collaborative and Dynamic Model of specialist and advanced nursing and midwifery practice. Therefore, the development of a schema of quality and performance metrics will track daily activities and align these with "what matters most to patients." The process will enable the grouping of outcomes by type into varying competencies/capabilities such as organisational, patient-related and performance-related. There is a need to generate transparent evidence of how nursing and midwifery contributes to high-quality care and health service performance. According to Maben et al. (2012), the development and implementation of nursing and midwifery metrics are part of that evidence.

\subsection{Self- and peer evaluation}

The self- and peer evaluation builds on competencies and allows for lifelong learning to occur within zones of complexity and levels of capability in a continuously changing healthcare context. A Likertstyle rating scale is located within the self- and peer evaluation element. This will facilitate assessment on the achievement of the core concepts as the specialist or advanced practitioner strives towards alignment of each within a given context. Thus, a practitioner may have these entire core concepts aligned at specialist level. But when population needs or an employment situation change occurs, disalignment can be accommodated. This is easily diagnosed through either selfassessment or peer assessment and measurement. In this context, the response on the part of the practitioner is proactive and preventive rather than reactive (Figure 1).

Insert Figure 1 here

\subsection{Education utility and continuous professional development}

Preparation of individuals for specialist and advanced practice roles needs to take cognisance of the totality of the elements of the model so that the specialist/advanced nurse/midwife practitioners are not only capable, but also educated to think scientifically. They can be part of a research culture, be partnered with patients and be able to inform patients about the evidence and lack of evidence thereof within an ethical decision-making framework. The vision of this model is that every nurse or midwife can use his or her knowledge and desire to continuously learn at a higher level, while actively engaged in practice and become a scholar-practitioner.

\subsection{Evaluation utility}

While a detailed explanation of the key components of this model is beyond the scope of this article, nevertheless, it is necessary to highlight its evaluative capacity. The various elements of this model can each be evaluated independently, in terms of role evaluation or service impact evaluation. Likewise, the overall model lends itself to an evaluative framework suitable for application in a range of clinical contexts. 


\section{CONCLUSION}

This model was developed from a critique of the extant literature and current models and is supported by the qualitative findings, albeit a small study. The model embraces capability as a concept that emphasises lifelong learning in uncertain as well as familiar intricate clinical situations.

The model recommends the implementation of key performance indicator schema and quality care metrics, alongside their evaluation by specialist and advanced nurse/midwife practitioners. This model recommends that the measurement of patient-reported outcomes, clinical outcomes and health economic outcomes by specialist and advanced nurse and midwife practitioners is required for accountability, that is, professional integrity.

It is anticipated that testing this Universal, Collaborative and Dynamic Model of specialist and advanced nursing and midwifery practice will provide a consensus definition as to what constitutes high-quality care, in the context of what matters most to patients/clients across their healthcare trajectory.

Specialist and advanced nurse/midwife practitioners must be fully integrated into emerging models of care that foster collaboration among healthcare providers from appropriate disciplines to meet patient's healthcare needs, across the lifespan. This interdisciplinary team approach does not mean that professional disciplines merge boundaries in a transdisciplinary way. Rather, nurse and midwifery management of episodes of care is seamless in a quantifiable and qualitative way.

\section{RELEVANCE TO CLINICAL PRACTICE}

The model design has Universal application in a range of contexts " $U$. " It is Collaborative in its inclusivity of all key stakeholders " $\mathrm{C}$." The model is Dynamic pertinent to accommodating movement of nurses and midwives across health continua rather than plateauing in very specialised "silos" "D." Furthermore, testing this Universal, Collaborative and Dynamic Model of specialist and advanced nursing and midwifery practice will be emancipatory in the context of capturing what precisely "matters most to patients" across diverse healthcare milieus.

\section{CONTRIBUTIONS}

Study design: $L O^{\prime} C, M C, R S, D O ' B, D O^{\prime} L$; data collection and analysis: $L O^{\prime} C, M C, R S, D O ' B, D O ' L$, MMcN, GF, MEG, AC; and manuscript preparation: LO'C, DO'L, MEG, AC.

\begin{tabular}{|l|l|}
\hline Demographic data & Number of participants \\
\hline Age range & 1 \\
\hline $\mathbf{3 0 - 3 5}$ & 0 \\
\hline $\mathbf{3 5 - 4 0}$ & 4 \\
\hline $\mathbf{4 0 - 4 5}$ & 5 \\
\hline $\mathbf{4 5 - 5 0}$ & \\
\hline
\end{tabular}




\begin{tabular}{|l|l|}
\hline $\mathbf{5 0 - 5 5}$ & 5 \\
\hline Gender & 2 \\
\hline Male & 13 \\
\hline Female & \multicolumn{2}{|l|}{} \\
\hline Highest level of education & 3 \\
\hline Postgraduate diploma & 10 \\
\hline Masters & 2 \\
\hline Doctorate & 8 \\
\hline Time in the role & \multicolumn{2}{|l}{} \\
\hline O-10 years & 3 \\
\hline Over 10 years & \\
\hline
\end{tabular}

Table 2. Roles of Participants

\begin{tabular}{|l|l|l|}
\hline Titles & Total number & $\begin{array}{l}\text { Participant Code } \\
\text { Identification }\end{array}$ \\
\hline Registered Advanced Nurse Practitioner & 4 & Identified as AP1-4 \\
\hline Nurse Specialist & 3 & \\
\hline Midwife Specialist & & Identified as CS1-3 \\
\hline Mental Health Specialist (Community) & & \\
\hline Policy, Registration and Management and Education & 5 & \\
\hline Medicine, Pharmacy and Nursing Representative Body & 3 & Identified as PRME1-5 \\
\hline Total & 15 & Identified as MPNRB1-3 \\
\hline
\end{tabular}

